\title{
Neutralising reactivity against SARS-CoV-2 B.1.617.2 (Delta) variant by vaccination status and pre-exposure
}

\section{Enrico Lavezzo ( $\nabla$ enrico.lavezzo@unipd.it)}

Department of Molecular Medicine, University of Padova, Padua, Italy https://orcid.org/0000-00023902-8356

\section{Monia Pacenti}

Azienda Ospedale Padova, Padova, Italy

\section{Laura Manuto}

Department of Molecular Medicine, University of Padova, Padua, Italy

\section{Caterina Boldrin}

Azienda Ospedale Padova, Padova, Italy

\section{Margherita Cattai}

Azienda Ospedale Padova, Padova, Italy

\section{Marco Grazioli}

Department of Molecular Medicine, University of Padova, Padua, Italy

\section{Federico Bianca}

Department of Molecular Medicine, University of Padova, Padua, Italy

\section{Margherita Sartori}

Department of Molecular Medicine, University of Padova, Padua, Italy

\section{Federico Caldart}

Gastroenterology Unit, Department of Medicine, Verona B. Roma University Hospital, Verona, Italy

\section{Gioele Castelli}

Department of Cardiac, Thoracic, Vascular Sciences and Public Health, University of Padova, Padova, Italy https://orcid.org/0000-0002-5925-4035

\section{Michele Nicoletti}

Department of Cardiac, Thoracic, Vascular Sciences and Public Health, University of Padova, Padova, Italy https://orcid.org/0000-0002-0233-9134

\section{Eleonora Nieddu}

Department of Surgery, Oncology and gastroenterology, University of Padova, Padova, Italy

\section{Elisa Salvadoretti}

Paediatrics Unit, Mother and Child Hospital, Surgery, Dentistry, Maternity and Infant Department, Verona University Hospital, Verona, Italy

\section{Beatrice Labella}


Neurology Unit, Department of Clinical and Experimental Sciences, University of Brescia, Brescia, Italy

\section{Ludovico Fava}

Department of Cardiac, Thoracic, Vascular Sciences and Public Health, University of Padova, Padova, Italy

\section{Maria Vanuzzo}

Azienda Ospedale Padova, Padova, Italy

\section{Vittoria Lisi}

Azienda Ospedale Padova, Padova, Italy

\section{Maria Antonello}

Department of Molecular Medicine, University of Padova, Padua, Italy

\section{Carmela Grimaldi}

Department of Molecular Medicine, University of Padova, Padua, Italy

\section{Chiara Zulian}

Azienda Ospedale Padova, Padova, Italy

\section{Claudia Del Vecchio}

Department of Molecular Medicine, University of Padova, Padova, Italy

\section{Mario Plebani}

Department of Medicine, University of Padova, Padova, Italy

\section{Andrea Padoan}

Department of Medicine, University of Padova, Padova, Italy

\section{Daniela Maria Cirillo}

Division of Immunology, Transplantation and Infectious Disease, IRCCS Ospedale San Raffaele, Milan, Italy https://orcid.org/0000-0001-6415-1535

\section{Alessandra Brazzale}

Department of Statistical Sciences, University of Padova, Padova, Italy

\section{Giovanni Tonon}

Center for Omics Sciences, IRCCS Ospedale San Raffaele

\section{Stefano Toppo}

1. Department of Molecular Medicine, University of Padua

\section{laria Dorigatti}

MRC Centre for Global Infectious Disease Analysis and Jameel Institute, School of Public Health, Imperial College London, London, United Kingdom

\section{Andrea Crisanti}

Department of Molecular medicine University of Padova

\section{Article}

\section{Keywords:}


Posted Date: December 8th, 2021

DOI: https://doi.org/10.21203/rs.3.rs-1131280/v1

License: (c) (i) This work is licensed under a Creative Commons Attribution 4.0 International License. Read Full License 
Neutralising reactivity against SARS-CoV-2 B.1.617.2 (Delta) variant by vaccination status and preexposure

3

Enrico Lavezzo ${ }^{1 \wedge}$, Monia Pacenti ${ }^{2 \wedge}$, Laura Manuto ${ }^{1 \wedge}$, Caterina Boldrin ${ }^{2}$, Margherita Cattai $^{2}$, Marco Grazioli $^{1}$, Federico Bianca ${ }^{1}$, Margherita Sartori ${ }^{1}$, Federico Caldart ${ }^{3}$, Gioele Castelli ${ }^{4}$, Michele Nicoletti ${ }^{4}$, Eleonora Nieddu ${ }^{5}$, Elisa Salvadoretti ${ }^{6}$, Beatrice Labella ${ }^{7}$, Ludovico Fava ${ }^{4}$, Maria Cristina Vanuzzo ${ }^{2}$, Vittoria Lisi ${ }^{2}$, Maria Antonello ${ }^{1}$, Carmela Ileana Grimaldi ${ }^{1}$, Chiara Zulian ${ }^{2}$, Claudia Del Vecchio $^{1}$, Mario Plebani ${ }^{8}$, Andrea Padoan $^{8}$, Daniela Cirillo ${ }^{9}$, Alessandra R Brazzale ${ }^{10}$, Giovanni Tonon ${ }^{11,12}$, Stefano Toppo ${ }^{1, *}$, Ilaria Dorigatti ${ }^{13, *}$ \& Andrea Crisanti ${ }^{1,2,14, *}$

${ }^{\wedge}$ Contributed equally

* Joint senior authors

${ }^{1}$ Department of Molecular Medicine, University of Padova, Padova, Italy

${ }^{2}$ Azienda Ospedale Padova, Padova, Italy

${ }^{3}$ Gastroenterology Unit, Department of Medicine, Verona B. Roma University Hospital, Verona, Italy

${ }^{4}$ Department of Cardiac, Thoracic, Vascular Sciences and Public Health, University of Padova, Padova, Italy ${ }^{5}$ Department of Surgery, Oncology and gastroenterology, University of Padova, Padova, Italy

${ }^{6}$ Paediatrics Unit, Mother and Child Hospital, Surgery, Dentistry, Maternity and Infant Department, Verona University Hospital, Verona, Italy

${ }^{7}$ Neurology Unit, Department of Clinical and Experimental Sciences, University of Brescia, Brescia, Italy ${ }^{8}$ Department of Medicine, University of Padova, Padova, Italy

${ }^{9}$ Emerging Bacterial Pathogens Unit, Division of Immunology, Transplantation and Infectious Diseases, IRCCS San Raffaele Scientific Institute, Milan, Italy

${ }^{10}$ Department of Statistical Sciences, University of Padova, Padova, Italy

${ }^{11}$ Center for Omics Sciences, IRCCS Ospedale San Raffaele, Milan, Italy

${ }^{12}$ Functional Genomics of Cancer Unit, Division of Experimental Oncology, IRCCS San Raffaele Scientific Institute, Milano, Italy

${ }^{13} \mathrm{MRC}$ Centre for Global Infectious Disease Analysis and Jameel Institute, School of Public Health, Imperial College London, London, United Kingdom

${ }^{14}$ Department of Life Sciences, Imperial College London, London, UK 
In February and March 2020, one of the first Italian clusters of SARS-CoV-2 infection was detected in the municipality of $\mathrm{Vo}^{\prime}$. Positive subjects were followed up at 2 and 9 months post-infection with different immuno-assays and a micro-neutralisation test. Here we report on the results of the third serosurvey conducted in the same population in June 2021,15 months post-infection, when we tested $61 \%$ of the infected individuals ( $n=76)$. Antibodies against the spike $(S)$ antigen significantly decreased $(P<0.006$, Kruskal-Wallis test) among unvaccinated subjects $(n=35)$ and increased $(P<0.0001)$ in vaccinated individuals $(n=41)$, whereas those against the nucleocapsid $(N)$ decreased in the whole cohort. From the comparison with two control groups (naïve Vo' inhabitants $(n=20)$ and healthcare workers ( $H C W, n=61)$ ), subjects vaccinated post exposure (hybrid immunity) had higher antibody levels $(P<0.0001)$ than subjects vaccinated when naïve. Two doses of vaccine elicited stronger anti-S antibody response than natural infection $(P<0.0001)$. Finally, the neutralising reactivity of sera against the B.1.617.2 (Delta) was lower than compared to the B.1 strain (median 1:320 versus 1:1280 1/dil, $P<0.0001$, and 1:640 versus 1:2560 1/dil, $\mathrm{P}=0.0014$, after one or two vaccine doses, respectively), although subjects with hybrid immunity maintained neutralising titres above 1:40 1/dil.

\section{Introduction}

Understanding the extent and duration of protection developed upon natural SARS-CoV-2 infections and vaccination is a current research priority. Evidence suggests that more than $90 \%$ of COVID-19 patients seroconvert after natural infection and develop variable levels of neutralising antibodies ${ }^{1-3}$, and demonstrates that the currently EMA and FDA approved vaccines induce humoral and cellular immunity in most individuals ${ }^{4-7}$. However, antibody titres have been reported to wane over time ${ }^{8}$. Although memory $B$ cells and cellular immunity can offer a quick and potent response in case of re-exposure to the virus ${ }^{9}$, preventing re-infections ${ }^{10-15}$ and offering long-term protection regardless of the presence of antibodyescaping mutations ${ }^{16-18}$, the interplay between antibody and cellular immunity, and the variation of naturally- and vaccine-acquired protection, remain to be fully characterised and understood. From an immunological perspective, there can be significant differences in the immune response generated by vaccines in individuals who were not exposed to SARS-CoV-2 before vaccination, and in subjects who recovered from a naturally acquired infection (so called 'hybrid immunity'). Recent studies have reported of increased potency of 'hybrid immunity', with viral antigen persistence in some tissues being hypothesised as a potential mechanism driving the process of memory B and T cell maturation, resulting in an increased affinity against viral antigens. Long term immunity against SARS-CoV-2 infection is jeopardized by the continuous evolution of the virus, 
escaping the immune protection elicited from infection and vaccination, thus posing further challenges for epidemic control. The most widespread variants currently circulating are called Delta plus strains ${ }^{19,20}$ and belong to a group of sub-lineages of the B.1.617.2 variant of concern (VOC), which emerged in India in October $2020^{21}$ and rapidly spread across the globe. To date, monitoring viral evolution is a central component of the epidemiological surveillance implemented in several countries to inform situation awareness and detect, new variants in local and global populations. While some VOCs with key changes in the spike protein were demonstrated to have a reduced susceptibility to neutralising antibodies ${ }^{22-25}$, there is no firm evidence that B.1.617.2 and its descendant sub-lineages have increased neutralisation resistance ${ }^{26}$, although some contrasting results emerged ${ }^{27}$. To investigate the interaction of natural immunity and vaccination in inducing protective immunity, in June 2021 we conducted a serological and viral neutralization study on a highly characterized cohort of subjects infected during the first wave, back in February 2020. This study follows on from the previous serosurveys conducted in the same population at two and nine months after the initial SARS-CoV-2 outbreak ${ }^{8,28}$, and provides unique longitudinal data on the magnitude, neutralizing ability, and persistence of the antibody response against the spike (S) and nucleocapsid $(\mathrm{N})$ antigens in unvaccinated pre-exposed subjects as well as vaccinated pre-exposed and naïve subjects, against both a B.1 SARS-CoV-2 strain circulating at the start of the pandemic and the currently circulating B.1.617.2 strain.

\section{Results}

\section{Serum reactivity to spike $(\mathrm{S})$ and nucleocapsid $(\mathrm{N})$ antigens}

In June 2021, 76 subjects infected by SARS-CoV-2 in February/March 2020 (as defined by the ground truth definition, see Methods) were tested with the same methods applied in the previous surveys (Methods) (Fig. 1). Overall, all 76 (100\%, 95\% Confidence Interval (CI) 95.3-100\%) individuals tested positive to at least one assay, with 9 (11.8\%, 95\% Cl 5.6-21.3\%) being positive to all three of them. As observed in our previous surveys, in June 2021 we observed strong differences in the proportion of positive subjects depending on the assay used, with $11.8 \%$ (9 out of $76,95 \% \mathrm{Cl} 5.6-21.3 \%$ ), $80.3 \%$ (61 out of $76,95 \% \mathrm{Cl} 69.5-88.5 \%$ ), and 93.4\% (71 out of 76, 95\% $\mathrm{Cl} 85.3-97.8 \%$ ) testing positive at the 15 months follow up for Abbott, DiaSorin and Roche, respectively. In June 2021, neutralising titres greater than 1:40 were found in $56.6 \%$ (43 out of 76, 95\% Cl 44.7-67.9\%) of subjects. Of additional 61 volunteering subjects, who took part in the June 2021 survey and were not identified as infected in February/March 2020 according to our ground truth definition, 3 had a positive swab between May and December 2020, and 8 showed positivity to at least two different serological assays and were excluded from the analyses; the remaining 50 subjects were used as a naïve control group. 
a Infected subjects in
Vo' up to April 2020

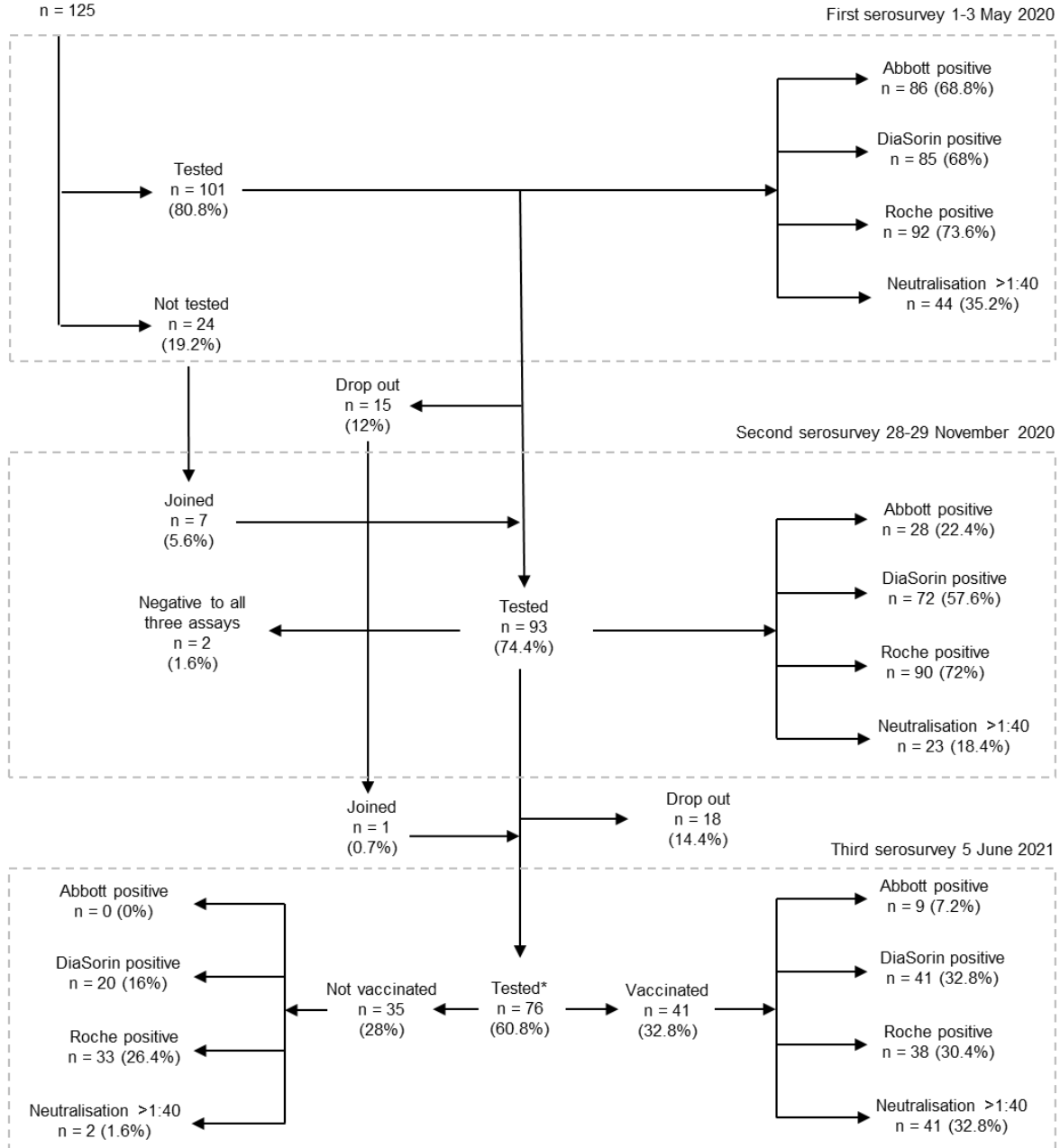

* Additional 61 subjects not belonging to the original ground truth were tested in June 2021

b

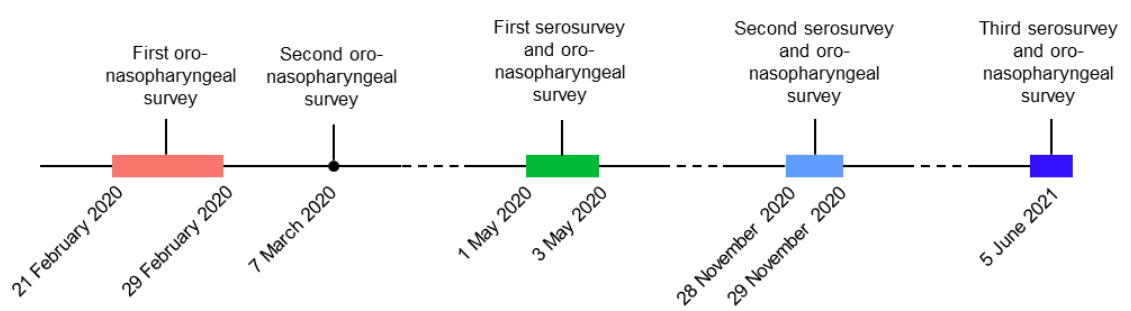

Fig. 1. Description of the study. a) Flow chart illustrating the study design, which focuses on the subjects who were found to be positive early in the pandemic (from February to May 2020, according to the ground truth definition). The serosurveys were conducted in Vo' on three time points, 1-3 May 2020, 28-29 November 2020, and 5 June 2021. b) Timeline of the surveys conducted in the study area since the start of the SARS-CoV-2 epidemic in Vo'. 
112 On $5^{\text {th }}$ June $2021,53.9 \%$ (41 out of $76,95 \% \mathrm{Cl} 42.1-65.5 \%$ ) of the participants previously infected by SARS-

113 CoV-2 according to the ground truth definition had received at least one dose of vaccine at least seven days

114 before testing. As expected, vaccination had a strong impact on S-targeting antibody levels (Fig. 2) but not

115 on those directed against the $\mathrm{N}$ antigen (Fig. 3). All vaccinated subjects showed reactivity against the $\mathrm{S}$

116 antigen, had a neutralising titre greater than 1:40 (41 out of 41 for both DiaSorin and neutralisation, $95 \% \mathrm{Cl}$

117 91.4-100\%) (Fig. 2b, 2d), and they still showed reactivity against the $\mathrm{N}$ antigen either when using Abbott

118 (22.0\%, 9 out of $41,95 \% \mathrm{Cl} 10.6-37.7 \%)$ or Roche (92.7\%, 38 out of $41,95 \% \mathrm{Cl} 80.1-98.4 \%)$ assays

119 (Supplementary Fig. $1 \mathrm{~b}$ and $1 \mathrm{~d}$ ). In the unvaccinated group the serum reactivity against the $\mathrm{S}$ antigen was

120 significantly lower compared to the vaccinated subjects, with positivity rates of $57.1 \%$ (20 out of $35,95 \% \mathrm{Cl}$

$12139.3-73.7 \%$ ) and 5.7\% (2 out of 35, 95\% Cl 0.7-19.2\%) for DiaSorin and microneutralisation assays,

122 respectively (Fig. 2 a-d). Among 50 naïve subjects, $37.0 \%$ (20 out of $54,95 \% \mathrm{Cl} 24.3-51.3 \%$ ) had received at

123 least one dose of vaccine at least seven days before testing. Of vaccinated naïve subjects, $0 \%$ ( 0 out of 20,

$12495 \% \mathrm{Cl} 0.0-16.9 \%$ ), $95.0 \%$ (19 out of $20,95 \% \mathrm{Cl} 75.1-99.9 \%$ ), $0 \%$ (0 out of $20,95 \% \mathrm{Cl} 0.0-16.9 \%$ ), and $25 \%$ (5

125 out of $20,95 \% \mathrm{Cl} 8.7-49.1 \%$ ) were positive to Abbott, DiaSorin, Roche and neutralisation, respectively,

126 whereas $8.8 \%$ (3 out of $34,95 \% \mathrm{Cl} 1.9-23.7 \%$ ), 35.3\% (12 out of $34,95 \% \mathrm{Cl}$ 19.8-53.5\%), $2.9 \%$ (1 out of 34 ,

$12795 \% \mathrm{Cl} 0.1-15.3 \%$ ), and $0 \%$ (0 out of $34,95 \% \mathrm{Cl} 0.0-10.3 \%$ ) of the unvaccinated and naïve (as of

128 February/March 2020) subjects showed positivity to the same tests. These latter among naïve unvaccinated

129 subjects are most likely false positives, since the percentages are in line with our previous positive

130 predictive values estimates for the different assays ${ }^{8}$ and the positivity to one test is never confirmed by any

131 of the others. 


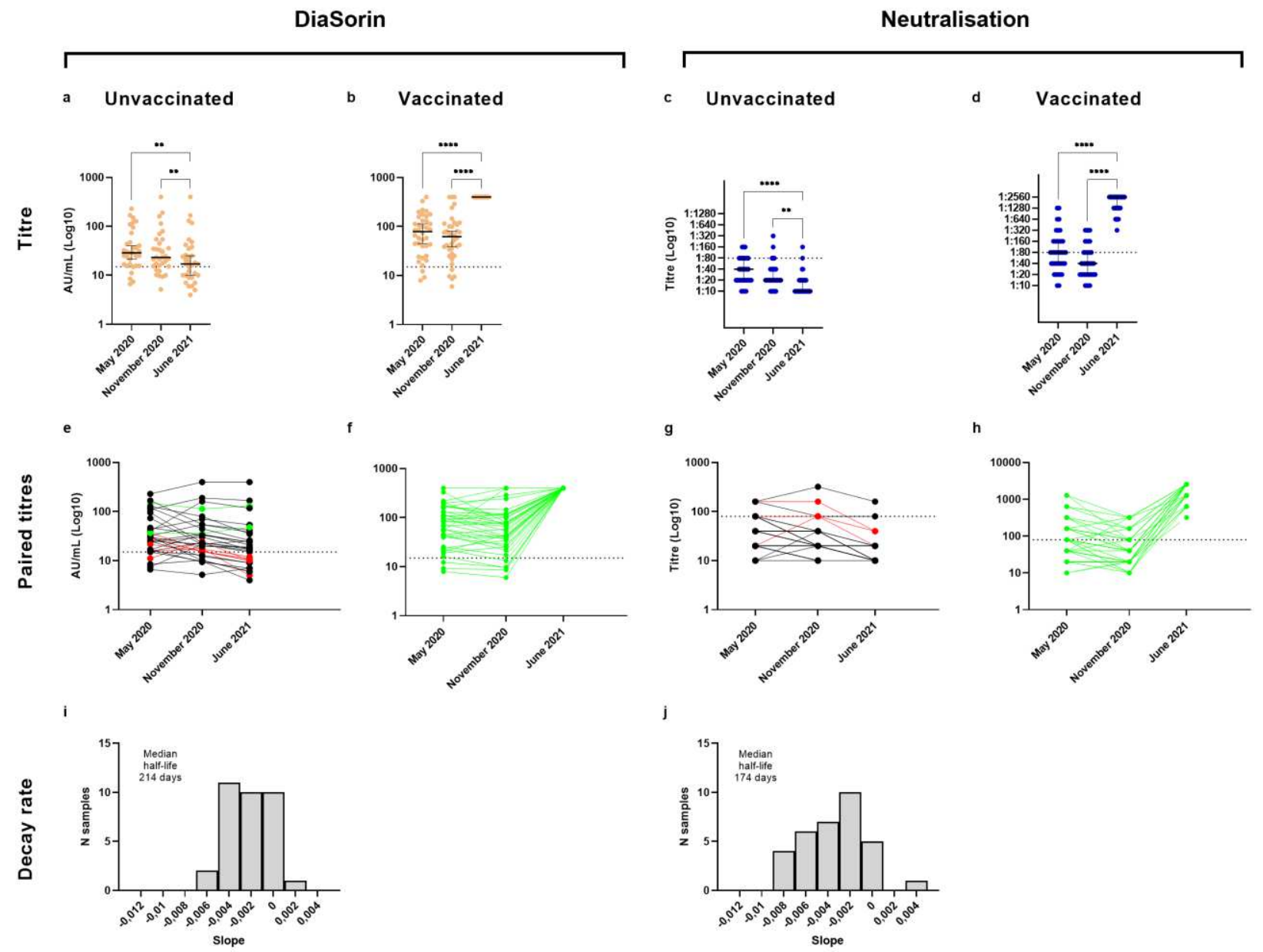

Fig. 2. Anti-S antibody titres and dynamics in vaccinated and unvaccinated subjects with pre-exposure to SARS-CoV-2. a-d) Observed antibody titres in unvaccinated and vaccinated subjects exposed to SARS-CoV-2 and tested in May 2020, November 2020 and June 2021 by DiaSorin (vaccinated n=38, P 0.0001 from November 2020 to June 2021; unvaccinated n=33, P = 0.0041 from November 2020 to June 2021) and micro-neutralisation assays (vaccinated $n=38, P<0.0001$ from November 2020 to June 2021; unvaccinated $n=32, P=0.0053$ from November 2020 to June 2021). The horizontal line represents the median, the vertical line represents the $95 \%$ confidence intervals. e-h) Observed individual-level paired antibody titres in subjects exposed to SARS-CoV-2 and tested in May 2020, November 2020 and June 2021. In June 2021, $60.6 \%$ ( 20 out of 33 unvaccinated subjects, $95 \% \mathrm{Cl} 42.1-77.1 \%$ ) and $6.3 \%$ (2 out of 32 unvaccinated individuals, $95 \% \mathrm{Cl} 0.8-20.8 \%$ ) had antibodies more than 15 months post infection according to DiaSorin and micro-neutralisation, respectively. Subjects with increasing titres are coloured in green, while subjects with a negative result in June 2021 are presented in red. i-j) Estimated antibody decay rate distributions calculated among the unvaccinated subjects exposed to SARS-CoV-2 in February/March 2020 and tested in May 2020, November 2020 and June 2021. We estimated a median half-life of 214 (95\% Cl 168-288) days and 174 (95\% $\mathrm{Cl} 146-202)$ days for the antibodies detected by the DiaSorin and micro-neutralisation assays, 


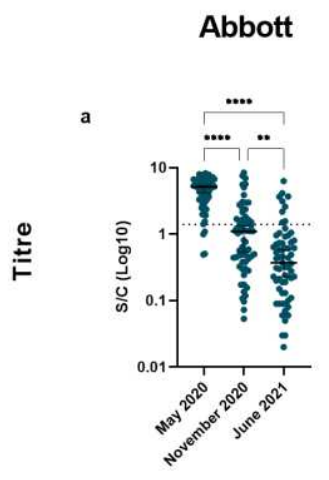

c
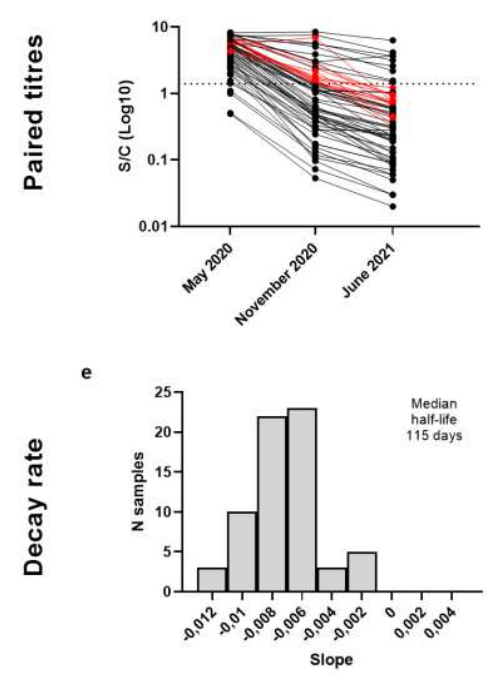

Roche

b
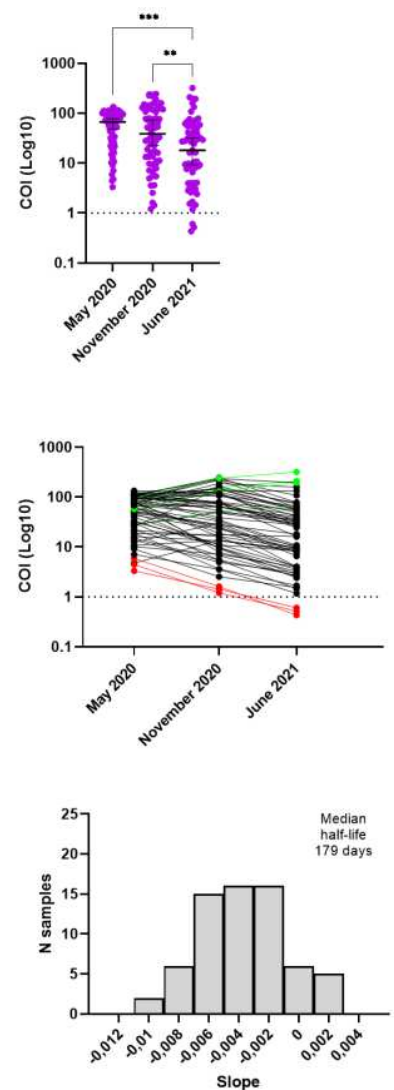

152

Fig. 3. Anti-N antibody titres and dynamics in subjects with pre-exposure to SARS-CoV-2.

a-b) Observed antibody titres in subjects exposed to SARS-CoV-2 and tested in May 2020, November 2020 and June 2021 with Abbott ( $n=65, P=0.0095$ from November 2020 to June 2021) and Roche assays ( $n=65$, $P=0.0073$ from November 2020 to June 2021). The horizontal line represents the median, the vertical line represents the $95 \%$ confidence intervals. c-d) Observed individual-level paired antibody titres in subjects exposed to SARS-CoV-2 and tested in May 2020, November 2020 and June 2021. In June 2021, 13.8\% (9 out of 65 subjects, $95 \% \mathrm{Cl} 6.5-24.7 \%$ ) and $95.4 \%$ (62 out of 65 subjects, $95 \% \mathrm{Cl} 87.1-99.0$ ) resulted positive to Abbott and Roche assays respectively, more than 15 months post infection. Subjects with increasing titres are coloured in green, and subjects with a negative result in June 2021 are presented in blue. e-f) Estimated antibody decay rate distribution calculated among subjects exposed to SARS-CoV-2 in February/March 2020 and tested in May 2020, November 2020, and June 2021. We estimated a median half-life of 115 (95\% $\mathrm{Cl} 105-126)$ days and 179 (95\% Cl 146-255) days for the antibodies detected by the Abbott and Roche 
assays, respectively. Asterisks indicate $* p<0.05, * * p<0.01, * * * p<0.001, * * * * p<0.0001$. Statistical significance of antibody levels was evaluated by Kruskal-Wallis test.

\section{Antibody dynamics in vaccinated and unvaccinated subjects}

Among vaccinated subjects, independently of the number of doses received, we found a significant increase of both DiaSorin and neutralisation titres (Wilcoxon matched-pairs signed rank test $p<0.0001$ for both cases), with all subjects showing an increasing trend (except three individuals who already had the maximum amount of antibodies quantifiable by DiaSorin); on the contrary, in the unvaccinated group, antibodies directed against the $\mathrm{S}$ antigen decreased significantly, as measured by DiaSorin and neutralisation (Wilcoxon matched-pairs signed rank test $p<0.0001$ for both cases) (Fig. 2). The serum reactivity against the $\mathrm{N}$ antigen progressively decreased with time irrespectively of the utilised assay in the whole cohort (Fig. 3, Wilcoxon matched-pairs signed rank test $p<0.001$ for both Abbott and Roche assays) and among vaccinated and unvaccinated individuals separately (Wilcoxon matched-pairs signed rank tests $p<0.001$ ) (Supplementary Fig. 1). Nonetheless, we observed a significant difference between anti-N antibody titres detected in June 2021 between vaccinated and unvaccinated individuals (Mann Whitney test $\mathrm{P}=0.0003$ and $\mathrm{P}=0.0005$ for Roche and Abbott, respectively) (Supplementary Fig. 2). Considering the subjects tested across all serosurveys conducted in May 2020,November 2020, and June 2021, the median half-life of the antibodies detected by Abbott, DiaSorin, Roche, and neutralisation are of 115 days (95\% Cl 105-126), 214 days (95\% Cl 168-288), 179 days (95\% Cl 146-255), and 174 (95\% Cl 146202) respectively.

\section{Correlation between two DiaSorin assays and neutralisation}

We assessed in parallel the performance of two DiaSorin tests, the first version targeting antibodies against the S1/S2 antigen and the updated version containing a full trimeric spike antigen. The two assays showed a strong correlation (Spearman's $r=0.820,95 \%$ Cl 0.670-0.906) (Supplementary Fig. 3) and concordance (Supplementary Table 1). We estimated a conversion factor between the two assays of $3.190(95 \% \mathrm{Cl}$ 3.061-3.319, $\mathrm{P}$ value $<0,0001$ ) by linear regression, and found high correlation between the antibody levels measured by the DiaSorin assays and the neutralising titres (DiaSorin S1/S2 vs neutralisation: Spearman's $r$ $=0.857,95 \%$ Cl 0.729-0.928; DiaSorin TrimericS vs neutralisation: Spearman's r = 0.752, 95\% Cl 0.5510.870; all $P$ values are significant, $P<0,0001$ ) (Supplementary Fig. 3).

\section{Hybrid immunity provides higher anti-S antibody and neutralisation titres than vaccination in naïve subjects}

We investigated the impact of past SARS-CoV-2 exposure to the humoral immune response induced by vaccination as measured by anti-S antibodies and neutralisation titres. Comparing the antibody titres of 
subjects from the Vo' cohort $(n=20)$ vaccinated when naïve to vaccinated individuals post exposure $(n=$ 41) we observed significantly higher titres in previously exposed individuals (Mann Whitney test, $P<$ $0.0001)$. Two vaccine doses reduced the observed difference in antibody titres between subjects vaccinated when naïve and subjects vaccinated post exposure (Fig. 4a, Kruskal-Wallis test, $\mathrm{P}=0.01$ ). Neutralisation and anti-S titres observed in pre-exposed vaccinated subjects after one and two vaccine doses were statistically comparable (Kruskal-Wallis test, $\mathrm{P}=1$ for both DiaSorin and neutralisation). Similar trends were observed when comparing the group of vaccinated subjects previously exposed to SARS-CoV-2 with an independent cohort of healthcare workers ( $\mathrm{HCW}, \mathrm{n}=61$ ) vaccinated when naïve from the complex operational unit (U.O.C.) of Microbiology and Virology of Padua University Hospital (Kruskal-Wallis test, $\mathrm{P}<0.0001$ for both DiaSorin and neutralisation)(Fig. 4b, 4d).

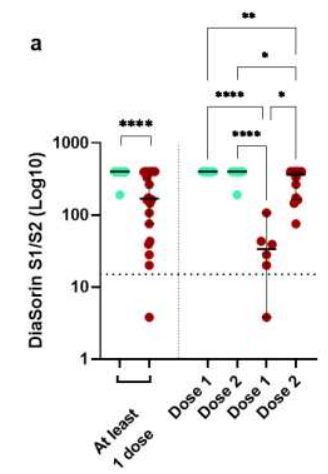

b
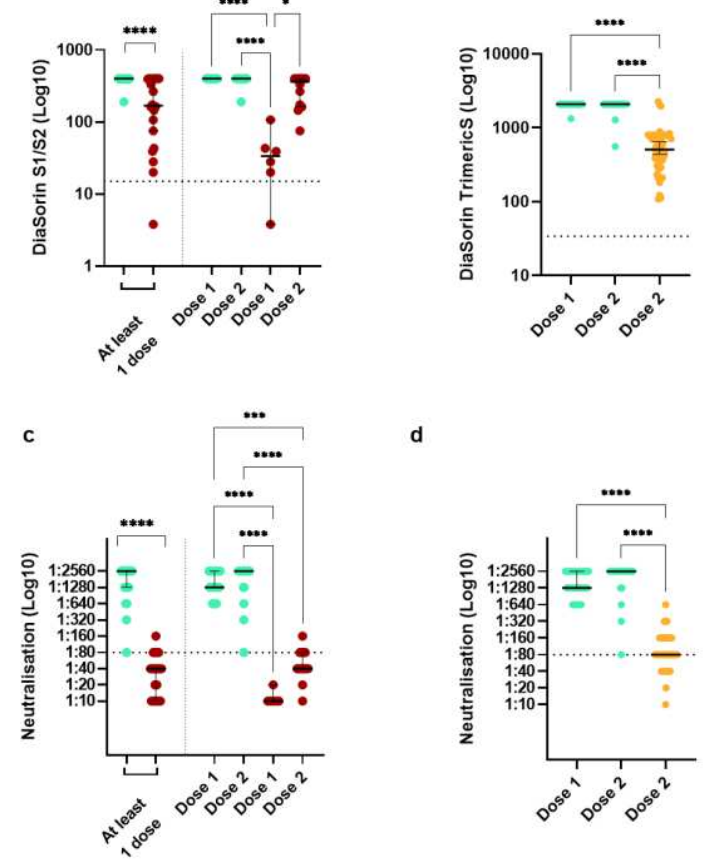

d

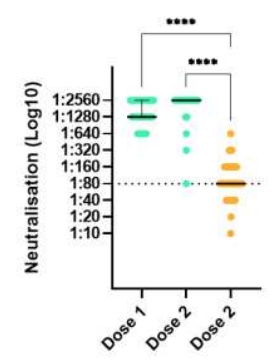

Fig. 4. Antibody levels in vaccinated naïve and vaccinated pre-exposed individuals according to DiaSorin and micro-neutralisation assays. a-b) Observed antibody levels measured by DiaSorin assays in vaccinated naïve and pre-exposed individuals with at least one dose of vaccine (Mann Whitney test, $\mathrm{P}<0.0001$ ) and with one or two doses of vaccine (Kruskal-Wallis test, vaccinated naïve versus pre-exposed subjects after one vaccine dose, $\mathrm{P}<0.0001$; after two vaccine doses, $\mathrm{P}=0.01$; vaccinated naïve HCW versus pre-exposed subjects after two vaccine doses, $P<0.0001)$. c-d) Observed neutralising antibody titres measured by a micro-neutralisation assay in vaccinated naïve and pre-exposed individuals with at least one dose of vaccine (Mann Whitney test, $\mathrm{P}<0.0001$ ) and with one or two doses of vaccine (Kruskal-Wallis test, vaccinated naïve 
versus pre-exposed subjects after one or two vaccine doses, $\mathrm{P}<0.0001$; vaccinated naïve HCW versus preexposed subjects after two vaccine doses, $P<0.0001)$. Asterisks indicate ${ }^{*} p<0.05,{ }^{*} p<0.01$, $* * * p<0.001, * * * * p<0.0001$. GT: ground truth, infected Vo' population; HCW: healthcare workers.

Two vaccine doses in naïve individuals trigger higher anti-S antibodies and neutralising titres than natural infection

Using the conversion factor calculated to convert the results of the old DiaSorin S1/S2 assay into the new DiaSorin trimericS assay, we compared the antibody response after natural infection with the response to vaccination, roughly two months after the immune stimulus. Vo' subjects exposed to SARS-CoV-2 in February/March 2020 and tested in May 2020 showed lower anti-S antibody levels with respect to both naïve subjects from Vo' (Kruskal-Wallis test, $\mathrm{P}<0.0001$ ) and HCW (Mann-Whitney test, $\mathrm{P}<0.0001$ ) after two doses of vaccine (Fig. 5a and 5b). A similar trend was observed for neutralising antibody titres, although the difference is significant only between exposed subjects and vaccinated HCW (Mann-Whitney test, $P=0.0002)$ (Fig. 5c and 5d).

a

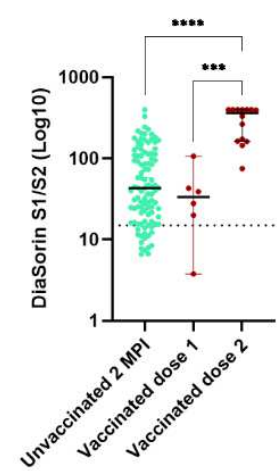

c

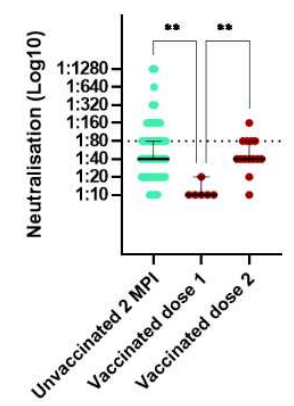

GT

Naive Vo' population

Naive $\mathrm{HCW}$ b

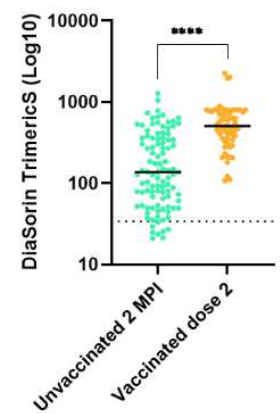

d

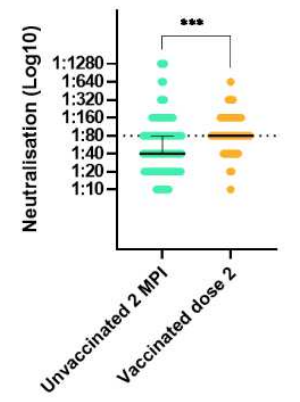

Fig. 5. Anti-S antibody levels and neutralisation titres induced by vaccination and natural infection. and HCW subjects vaccinated when naïve, according to (a) DiaSorin S1/S2 (Kruskal-Wallis test, 
unvaccinated pre-exposed versus vaccinated when naïve after one dose of vaccine, $P=1$, or two doses of vaccine, $\mathrm{P}<0.0001$ ), (b) DiaSorin TrimericS (Mann-Whitney test, unvaccinated pre-exposed versus HCW subjects vaccinated when naïve, $P<0.0001$ ) (c-d) and micro-neutralisation (Kruskal-Wallis test, unvaccinated pre-exposed versus vaccinated when naïve after one dose of vaccine, $P=0.001$, or two doses of vaccine, $\mathrm{P}=1$; Mann-Whitney test, unvaccinated pre-exposed versus HCW subjects vaccinated when naïve, $\mathrm{P}=0.0002$ ) assays. Asterisks indicate ${ }^{*} \mathrm{p}<0.05,{ }^{* *} \mathrm{p}<0.01,{ }^{* * *} \mathrm{p}<0.001, * * * \mathrm{p}<0.0001$. GT: ground truth, infected Vo' population; HCW: healthcare workers.

\section{Association analysis}

Among infected unvaccinated subjects, we observed no significant differences in the antibody titres by symptom occurrence, hospitalisation, sex, age-group and BMI. In the Vo' cohort, we observed no statistically significant difference in the number of vaccinated and not vaccinated subjects by infection status (according to the baseline ground truth definition), symptom occurrence and sex.

\section{Neutralisation reactivity of Delta VOC}

The sera obtained from vaccinated individuals pre-exposed to SARS-CoV-2 infection and subjects vaccinated when naïve were tested in a micro-neutralisation assay against the B.1.617.2 variant, to assess the neutralising ability of the humoral immunity mounted upon vaccination. Lower neutralising titres against the B.1.617.2 than compared to the B.1 strain were observed in individuals vaccinated after natural exposure, both after one and two vaccine doses (Kruskal-Wallis test, $\mathrm{P}<0.0001$ and $\mathrm{P}=0.0014$, respectively), although all but one subject maintained a neutralisation titre $>1: 40$ (1/dil) (20 out of 20 after one dose of vaccine, $100 \%, 95 \% \mathrm{Cl} 83.2-100 \%, 19$ out of 20 after two doses of vaccine, $95 \%, 95 \% \mathrm{Cl} 75.1$ 99.9\%)(Fig. 6a). The decrease in neutralisation caused by the B.1.617.2 variant was observed also in unvaccinated individuals previously exposed (Fig. 6b) (Mann Whitney test, $P=0.0002$ ), but in a context where most of them displayed low neutralising titres also against the B.1 strain (33 out of $35(94.3 \%, 95 \% \mathrm{Cl}$ 80.8-99.3\%) and 34 out of 35 (97.1\%, 95\% Cl 85.1-99.9\%) subjects with neutralising titres below 1:80 (1/dil) threshold against the B.1 and B.1.617.2 variants, respectively). A similar but non-significant trend was present in subjects vaccinated when naïve (Fig. 6c) (Kruskal-Wallis test, $\mathrm{P}=1$ and $\mathrm{P}=0.15$ after one or two vaccine doses, respectively). 


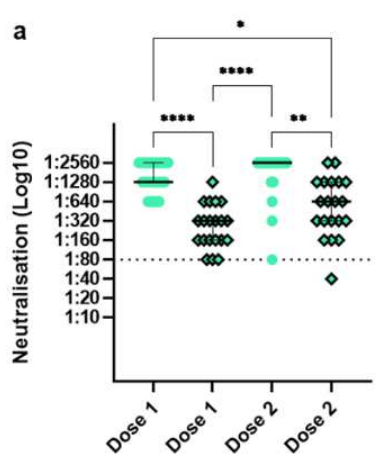

b

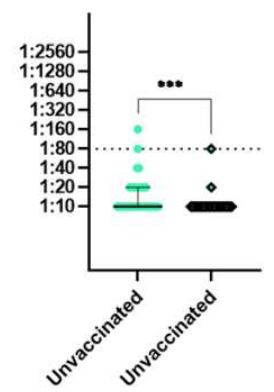

c

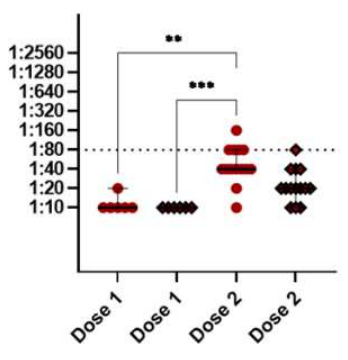

- B.1

ه B.1.617.2

266

Fig. 6. Neutralisation titres against the B.1 and B.1.617.2 SARS-CoV-2 variants in individuals vaccinated naïve or pre-exposed and unvaccinated pre-exposed subjects. a-b) Neutralising antibody titres against B.1 and B.1.617.2 SARS-CoV-2 variants among pre-exposed a) vaccinated and b) unvaccinated individuals (Kruskal-Wallis test, pre-exposed subjects after one $(P<0.0001)$ or two $(P=0.0014)$ doses of vaccine; Mann Whitney test, pre-exposed unvaccinated $\mathrm{P}=0.00002)$. c) Neutralising antibody titres against B.1 and Delta SARS-CoV-2 variants among vaccinated naive individuals (Kruskal-Wallis test, vaccinated naïve after one (P $=1)$ or two $(P=0.15)$ doses of vaccine). Asterisks indicate $* p<0.05, * * p<0.01, * * * p<0.001, * * * * p<$ 0.0001. GT: ground truth, infected Vo' population.

\section{Discussion}

Monitoring the serological response to SARS-CoV-2 infection and vaccination over time is crucial to estimate the persistence of circulating antibodies, their neutralising efficacy, and to inform vaccination policies. Due to the continuous emergence of new viral variants ${ }^{21}$, it is critical to assess the extent to which previous immunity, developed from natural infection or vaccination, protects against the new circulating strains.

The Vo' cohort is a highly characterised population including a core of individuals identified as exposed to SARS-CoV-2 back in February/March 2020, which has been followed-up through time in sequential swab and serological surveys until June 2021, roughly 15 months after viral exposure, thus offering unique insights into the long term antibody dynamics. The results presented in this study confirm the trends observed in our previous follow-up, performed at 9 months since the first wave in $\mathrm{Vo}^{\prime 8}$, with strong variability observed among serological tests, especially for the two assays targeting the $\mathrm{N}$ viral antigen. Of the identified SARS-CoV-2 cases who acquired the infection in February/March 2020 , only $11.8 \%(95 \% \mathrm{Cl}$ 5.6-21.3\%) tested positive by Abbott while 93.4\% (95\% Cl 85.3-97.8\%) tested positive by Roche after 15 months. This discrepancy could be due to differences in the employed antigens and to the fact that the $\mathrm{N}$ epitopes recognised by antibodies might change with time. In perspective, given that these two tests could 
allow to discern recent from past infections, they could be employed in future seroprevalence studies to assess the attack rate in vaccinated subjects and thus provide new data on the frequency of breakthrough infections as well as re-infection.

We found that all individuals infected at the start of the pandemic and tested 15 months later are positive to at least one serological assay, although the decreasing trend of antibody levels against both $\mathrm{S}$ and $\mathrm{N}$ antigens is confirmed, independently from the type of test used. In the absence of vaccination, the neutralising titres of the infected subjects drop almost completely below the 1:80 (1/dil) threshold. While the observed decrease in antibody titres is in line with other recent reports, it does not necessarily translate into an impaired immunity in these subjects, since humoral response is one arm of the adaptive immune response, which also includes cellular immunity and reactivation upon stimulation of memory $B$ and T cells $\mathrm{s}^{14,30,31}$.

Unexpectedly, we found a significant difference in the amount of circulating $\mathrm{N}$ targeting antibodies between vaccinated and unvaccinated subjects pre-exposed to SARS-CoV-2. To investigate this pattern we retrospectively analysed the differences in $\mathrm{N}$ targeting antibodies present in the two groups in the November serosurvey, before the beginning of the vaccination campaign. We found that the two groups are significantly different in terms of age, with vaccinated subjects being older than unvaccinated subjects (Suppl. fig. 2e). The age difference can be explained by the vaccination strategy and agrees with our previous observation that antibody levels were higher with increasing age in this cohort ${ }^{8}$. We found that the response to vaccination is different among subjects vaccinated after pre-exposure and when naïve: while a marked increase in S-targeting antibodies is observed in all individuals, antibodies induced by vaccination are higher in pre-exposed subjects. In vaccinated pre-exposed subjects, a single dose of vaccine saturates the dynamic range of DiaSorin assay and is shown to boost a strong neutralisation response, as confirmed in other studies ${ }^{32,33}$. This suggests that a single dose of vaccine in pre-exposed patients induces a robust immune response in support of the vaccination strategy implemented in Germany, France, Italy, and Israel among other countries. It has been shown that B cell maturation due to somatic hypermutation, possibly stimulated by long-term persistence of viral antigens in specific body niches ${ }^{9,34-36}$, can produce stronger and more specific antibodies ${ }^{37}$. By comparing the antibody levels in vaccinated naïve subjects (in June 2021) with those of patients who recovered from natural infection in May 2020, we demonstrate that a complete vaccination course confers stronger immunity than natural infection alone, at least in terms of serum antibodies as detected by both DiaSorin and neutralisation.

We tested the ability of antibodies developed against SARS-CoV-2 strains circulating early in the pandemic to neutralise the Delta variant of concern (VOC B.1.617.2), which is characterised by several mutations in the spike protein and an increased transmissibility that allowed this variant to become prevalent worldwide ${ }^{38}$. We observed a decrease in neutralising reactivity across all immunity profiles (naturally 
exposed and unvaccinated, vaccinated pre-exposed and vaccinated naïve). The reduction in neutralising reactivity is more evident in the vaccinated pre-exposed subjects (Fig. 6a), despite the neutralising titres remained above 1:40 (1/dil). Since neutralising IgG antibodies are the best current indication for protection against reinfection and correlate well with virological response and survival ${ }^{17,39}$, this finding is of particular importance in consideration of the efforts and resources that have been invested in the vaccination campaign in Italy and worldwide. Our results show that the vaccines currently deployed in Europe, although developed on a viral strain that is no longer circulating, are conferring strong and durable protection against the most prevalent strain (as of October 2021). These results confirm that vaccination is a safe and effective strategy to generate immunity against SARS-CoV-2. At the same time, it is critical to maintain and strengthen epidemiological and genomic surveillance, to monitor the potential emergence of new, immune-escaping variants in the future.

\section{Methods}

\section{Ethical approval statement}

All the serosurveys of the Vo' population were approved by the Ethics Committee for Clinical Research of the province of Padova (May survey approved on 30th April 2020, protocol number 0026971; November survey and additional follow up approved on 11th November 2020, protocol number 0068830). Study participation was by consent. For participants under 18 years of age, consent was provided by a parent or legal guardian.

\section{Laboratory methods}

\section{Oro-nasopharyngeal swabs}

Swab test were performed as previously described ${ }^{8,28}$. Briefly, swabs were inserted into the posterior pharynx first, rubbed over tonsillar pillars and posterior oropharynx and then over the nasal wall in the nostrils. SARS-CoV-2 genome was searched with an in-house real-time RT-PCR method targeting the envelope gene (E), according to Corman et al. ${ }^{40}$

\section{Serum antibodies detection}

IgG anti-SARS-CoV-2 were searched in venous blood collected in $5 \mathrm{ml} \mathrm{BD} \mathrm{Vacutainer} \mathrm{Serum} \mathrm{Separation}$ Tubes (SST) and centrifuged for $10 \mathrm{~min}$ at 1000-1300 RCF (g). Serological tests were performed by trained laboratory staff using the same commercial kits employed in previous serosurveys ${ }^{8}$ and produced by Abbott $^{41}$, DiaSorin ${ }^{42}$, and Roche ${ }^{43}$, applying the detection thresholds provided by the manufacturer (Table 1). For DiaSorin, both the new TrimericS and the previous S1/S2 kits were used for comparison.

\section{Micro-neutralisation assay}


Two independent assays were set up in parallel to assess the neutralisation ability of patients' seric antibodies against two viral isolates, a third passage B.1 strain isolated in March 2020 (GenBank accession MW468415) and a third passage B.1.617.2 strain from August 2021 (GenBank accession ...(waiting for the release of the accession number)). Heat-inactivated serum samples $\left(30 \mathrm{~min}\right.$ at $\left.56^{\circ} \mathrm{C}\right)$ were diluted 1:10 with DMEM FBS Free medium and filtered ( $0.22 \mu \mathrm{m}$ pore size). $50 \mu \mathrm{l}$ of viral isolate, diluted in DMEM FBS Free to the final concentration of 100 median tissue culture infective dose (TCID50), were mixed with an equal volume of two-fold serial dilutions of sera in 96-wells microplates and incubated for $1 \mathrm{~h}$ at $37^{\circ} \mathrm{C}$ in a humidified atmosphere with $5 \% \mathrm{CO}$. Following incubation, $100 \mu \mathrm{L}$ of VERO E6 cells suspended in DMEM $6 \% \mathrm{FBS}$ were added to each well and incubated at $37^{\circ} \mathrm{C}$. After $72 \mathrm{~h}$, cytopathic effect was assessed; the supernatant was removed and $120 \mu \mathrm{l}$ of $5 \%$ formaldehyde Gram's crystal violet $40 \% \mathrm{~m} / \mathrm{v}$ were added to each well, followed by 30 min of incubation. After a washing step with water, plates were allowed to dry and the absorbance was read at $595 \mathrm{~nm}$. The neutralisation titre was determined as the highest serum dilution showing an optical density (OD) of $90 \%$ or more with respect to the control sera.

\section{Definition of COVID-19 recovered patients (ground truth, GT)}

Multiple rounds of mass testing, that included oropharyngeal swabs and serological assays, allowed for the identification of all the residents in the municipality of Vo' who were infected and recovered from SARSCoV-2 infection during the first wave, between February and March 2020. To be included among COVID-19 recovered individuals, one of the following criteria had to be satisfied: i) a positive swab, ii) a viral neutralization titre greater than 1:40, or iii) serum reactivity against two serological tests with different antigen targets. We refer to this group as baseline ground truth (GT). It included 125 subjects, a size that perfectly fitted the seroprevalence estimated through a multinomial likelihood model ${ }^{8}$. These subjects were followed up at several time points to monitor the presence and persistence of antibodies against both the spike (S) and the nucleocapsid (N) antigens (Figure 1), as well as to investigate the presence of virus neutralising antibodies (Tables 1 and 2). We previously reported that all subjects belonging to the GT were positive to at least one serological assay in May 2020, about two months after the time of their infection (Fig. 1). On occasion of a second serological survey conducted in November $98.8 \%$ of GT subjects were still positive nearly 9 months after the infection ${ }^{8}$, although with strong differences depending on the test.

Table 1. Commercial assays employed in the study to identify IgG anti-SARS-CoV-2 antibody levels.

\begin{tabular}{ccccc}
\hline Test & Manufacturer & Recognised antigen & Method & Manufacturers' thresholds \\
\hline LIAISON ${ }^{\circledR}$ & DiaSorin & S1/S2 & CLIA $^{\text {a }}$ & Negative: $<12.0 \mathrm{AU} / \mathrm{mL}$ \\
SARS-CoV-2 S1/S2 & & & Equivocal: $12.0 \leq x<15.0 \mathrm{AU} / \mathrm{mL}$ \\
IgG & & & \\
\hline
\end{tabular}


Positive: $\geq 15.0 \mathrm{AU} / \mathrm{mL}$

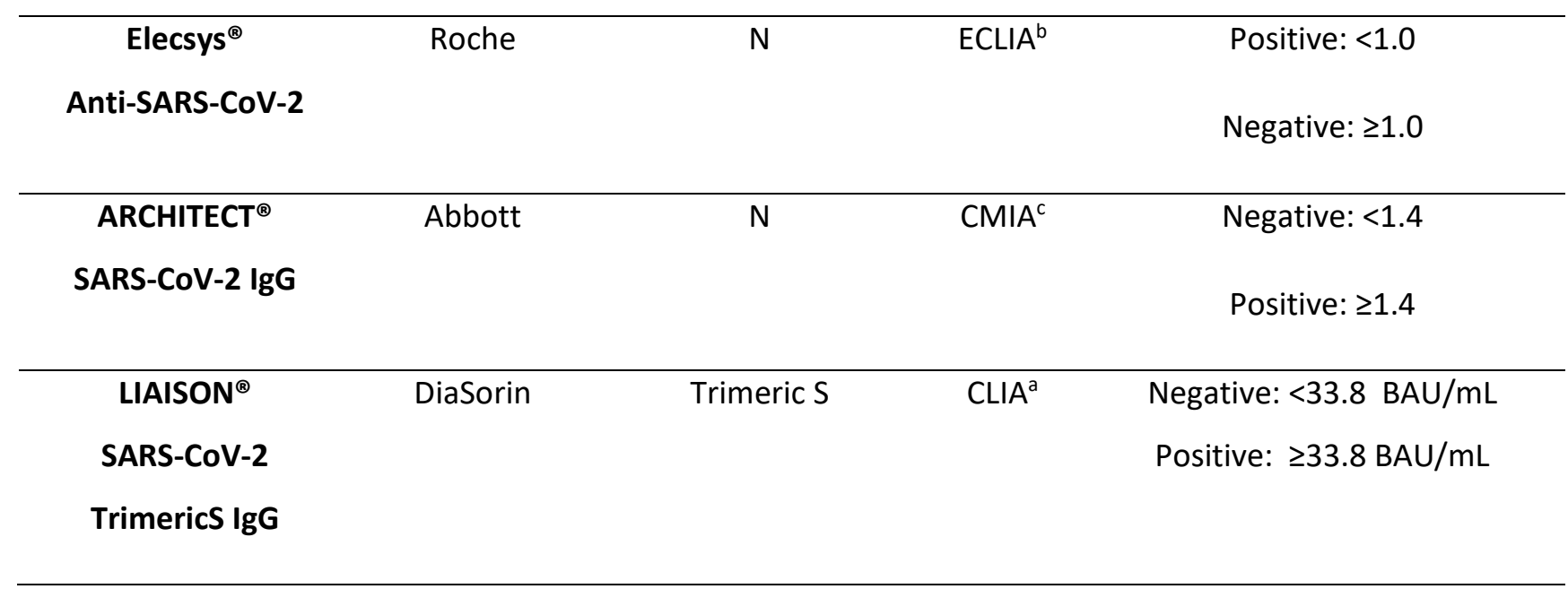

Table 2. Observed positivity rates by assays across the three serosurveys, stratified by vaccination status and dose for June 2021.

\begin{tabular}{|c|c|c|c|c|c|c|}
\hline \multirow[t]{2}{*}{ Test } & \multirow{2}{*}{$\begin{array}{l}\text { Detected } \\
\text { antigen }\end{array}$} & \multirow{2}{*}{$\begin{array}{c}\text { Positive } \\
\text { May } 2020 \\
\text { (\%) }\end{array}$} & \multirow{2}{*}{$\begin{array}{c}\text { Positive } \\
\text { November } \\
2020(\%)\end{array}$} & \multicolumn{3}{|c|}{ Positive June 2021 (\%) } \\
\hline & & & & $\begin{array}{c}\text { Not vaccinated } \\
(\%)\end{array}$ & 1 dose (\%) & 2 doses (\%) \\
\hline Abbott & $\mathrm{N}$ & $86 / 92(93,5)$ & $28 / 93(30,1)$ & $0 / 76(0)$ & $3 / 76(3,9)$ & $6 / 76(7,9)$ \\
\hline DiaSorin & $\mathrm{S}$ & $\begin{array}{c}85 / 101 \\
(84,2)\end{array}$ & $72 / 93(77,4)$ & $20 / 35(57,1)$ & $21 / 21(100)$ & 20/20 (100) \\
\hline Roche & $N$ & $92 / 92$ (100) & $90 / 93(96,8)$ & $33 / 76(43,4)$ & $19 / 76(25,0)$ & $19 / 76(25,0)$ \\
\hline Neutralisation & $\mathrm{S}$ & $44 / 98(44,9)$ & $23 / 93(24,7)$ & $2 / 35(5,7)$ & $21 / 21(100)$ & $20 / 20(100 \%)$ \\
\hline
\end{tabular}

Statistical methods

Estimates of antibody decay rate and association analysis

The antibody decay rate was estimated at the individual level as the logarithmic change in antibody values observed between May 2020 and June 2021 (within the same subject) divided by the number of days between the two serosurveys (400 days). The antibody half-life was estimated as the natural logarithm of 0.5 divided by the antibody decay rate, and was calculated on all subjects testing positive in May 2020 serosurvey and without doubling antibody levels in November 2020 and June 2021 (Abbott n=65, DiaSorin $n=29$, Roche $n=53$, neutralisation $n=9$ ). 
405 The associations between antibody levels and symptom occurrence, hospitalisation, sex, age-group and $406 \mathrm{BMI}$ and between vaccination and pre-exposure, symptom occurrence and sex were assessed using the 407 Kruskal-Wallis test. We used Fisher's exact test to assess the association between vaccination and previous 408 hospitalisation.

409

Data availability statement

411 The dataset is available at https://github.com/MedCompUnipd/Vo-Serology.git

412 Ethical approval statement

413 The third serosurvey of the Vo' population was approved by the Ethics Committee for Clinical Research of 414 the province of Padova. Study participation was by consent. For participants under 18 years of age, consent 415 was provided by a parent or legal guardian.

\section{Competing interests}

417 The authors declare no competing interests.

\section{Correspondence}

419 Please address correspondence to Prof Enrico Lavezzo (enrico.lavezzo@unipd.it) or Prof Andrea Crisanti 420 (andrea.crisanti@unipd.it).

\section{Author contributions}

422 Study conceptualisation: EL, AC, ID.

423 Coordination of data collection and curation: EL.

424 Performed laboratory testing: MP, CB, MC, CDV, MCV, VL, MA, IG, CZ, MP, AP.

425 Sampling logistics and collection: EL, FC, GC, MN, EN, ES, BL, LF, LM, MG, FB, MS.

426 Performed swab and blood sampling: FC, GC, MN, EN, ES, BL, LF.

427 Statistical analysis: EL, LM, ID, ST, ARB.

428 Funding acquisition: EL, ST, ID, GT, AC.

429 Methodology: EL, LM, ST, ID, ARB.

430 Visualisation: EL, LM, ID.

431 Writing - original draft: EL.

432 Writing - review \& editing: EL, ID, LM, ST, GT, ARB, AC. 


\section{Acknowledgments}

We thank the population of Vo' who participated to multiple serosurveys follow up. We acknowledge Abbott, DiaSorin and Roche Diagnostics for kindly supplying reagents, with no influence on the study design and data analysis. This work was supported by the Veneto Region. G.T. and A.C acknowledge Fondazione Umberto Veronesi, Misura Ricerca Covid 19, year 2020. E.L. and S.T. acknowledge research funding from the European Union's Horizon 2020 research and innovation programme, under grant agreement No 874735 (VEO). E.L. acknowledges funding from the University of Padova and the Department of Molecular Medicine (STARS-CoG ISS-MYTH and PRID/SID 2020). S.T. acknowledges funding from the University of Padua (TOPP_PRIV20_01 and TOPP_SID19_01). ID acknowledges research funding from a Sir Henry Dale Fellowship funded by the Royal Society and Wellcome Trust [grant 213494/Z/18/Z], the Abdul Latif Jameel Institute for Disease and Emergency Analytics, funded by Community Jameel and the MRC Centre for 449 450

\section{References}

1. Post, N. et al. Antibody response to SARS-CoV-2 infection in humans: A systematic review. PLOS ONE 15, e0244126 (2020).

2. Humoral response and PCR positivity in patients with COVID-19 in the New York City region, USA: an

3. Wu, J. et al. SARS-CoV-2 infection induces sustained humoral immune responses in convalescent patients following symptomatic COVID-19. Nat. Commun. 12, 1813 (2021). 
6. Folegatti, P. M. et al. Safety and immunogenicity of the ChAdOx1 nCoV-19 vaccine against SARS-CoV-2: a preliminary report of a phase $1 / 2$, single-blind, randomised controlled trial. The Lancet 396, 467-478 (2020).

7. Self, W. H. Comparative Effectiveness of Moderna, Pfizer-BioNTech, and Janssen (Johnson \& Johnson) Vaccines in Preventing COVID-19 Hospitalizations Among Adults Without Immunocompromising Conditions - United States, March-August 2021. MMWR Morb. Mortal. Wkly. Rep. 70, (2021).

8. Dorigatti, I. et al. SARS-CoV-2 antibody dynamics and transmission from community-wide serological testing in the Italian municipality of Vo'. Nat. Commun. 12, 4383 (2021).

9. Gaebler, C. et al. Evolution of antibody immunity to SARS-CoV-2. Nature 591, 639-644 (2021).

10. Hall, V. J. et al. SARS-CoV-2 infection rates of antibody-positive compared with antibody-negative health-care workers in England: a large, multicentre, prospective cohort study (SIREN). The Lancet 397, 1459-1469 (2021).

11. Hanrath, A. T., Payne, B. A. I. \& Duncan, C. J. A. Prior SARS-CoV-2 infection is associated with protection against symptomatic reinfection. J. Infect. 82, e29-e30 (2021).

12. Abu-Raddad, L. J. et al. SARS-CoV-2 antibody-positivity protects against reinfection for at least seven months with 95\% efficacy. EClinicalMedicine 35, (2021).

13. Hansen, C. H., Michlmayr, D., Gubbels, S. M., Mølbak, K. \& Ethelberg, S. Assessment of protection against reinfection with SARS-CoV-2 among 4 million PCR-tested individuals in Denmark in 2020: a population-level observational study. The Lancet 397, 1204-1212 (2021).

14. Hartley, G. E. et al. Rapid generation of durable B cell memory to SARS-CoV-2 spike and nucleocapsid proteins in COVID-19 and convalescence. Sci. Immunol. 5, eabf8891 (2020).

15. Chia, W. N. et al. Dynamics of SARS-CoV-2 neutralising antibody responses and duration of immunity: a longitudinal study. Lancet Microbe 2, e240-e249 (2021).

16. Reynolds, C. J. et al. Prior SARS-CoV-2 infection rescues B and T cell responses to variants after first vaccine dose. Science $372,1418-1423$ (2021).

17. Cromer, D. et al. Prospects for durable immune control of SARS-CoV-2 and prevention of reinfection. Nat. Rev. Immunol. 21, 395-404 (2021). 
18. Impact of SARS-CoV-2 variants on the total CD4+ and CD8+ T cell reactivity in infected or vaccinated individuals: Cell Reports Medicine. https://www.cell.com/cell-reports-medicine/fulltext/S26663791(21)00204-

4?_returnURL=https\%3A\%2F\%2Flinkinghub.elsevier.com\%2Fretrieve\%2Fpii\%2FS2666379121002044\%3F showall\%3Dtrue.

19. SARS-CoV-2 variants of concern as of 21 October 2021. European Centre for Disease Prevention and Control https://www.ecdc.europa.eu/en/covid-19/variants-concern.

20. SARS-CoV-2 variants of concern and variants under investigation. 43.

21. Tracking SARS-CoV-2 variants. https://www.who.int/emergencies/emergency-health-kits/traumaemergency-surgery-kit-who-tesk-2019/tracking-SARS-CoV-2-variants.

22. Wang, P. et al. Antibody resistance of SARS-CoV-2 variants B.1.351 and B.1.1.7. Nature 593, 130135 (2021).

23. Planas, D. et al. Sensitivity of infectious SARS-CoV-2 B.1.1.7 and B.1.351 variants to neutralizing antibodies. Nat. Med. 27, 917-924 (2021).

24. Wang, P. et al. Increased Resistance of SARS-CoV-2 Variant P.1 to Antibody Neutralization. 2021.03.01.433466 https://www.biorxiv.org/content/10.1101/2021.03.01.433466v2 (2021) doi:10.1101/2021.03.01.433466.

25. Zhou, D. et al. Evidence of escape of SARS-CoV-2 variant B.1.351 from natural and vaccine-induced sera. Cell 184, 2348-2361.e6 (2021).

26. Arora, P. et al. Delta variant (B.1.617.2) sublineages do not show increased neutralization resistance. Cell. Mol. Immunol. 1-3 (2021) doi:10.1038/s41423-021-00772-y.

27. Mlcochova, P. et al. SARS-CoV-2 B.1.617.2 Delta variant replication and immune evasion. Nature 16 (2021) doi:10.1038/s41586-021-03944-y.

28. Lavezzo, E. et al. Suppression of a SARS-CoV-2 outbreak in the Italian municipality of Vo'. Nature $584,425-429(2020)$.

29. Rogliani, P., Chetta, A., Cazzola, M. \& Calzetta, L. SARS-CoV-2 Neutralizing Antibodies: A Network Meta-Analysis across Vaccines. Vaccines 9, 227 (2021). 
30. Goel, R. R. et al. mRNA vaccines induce durable immune memory to SARS-CoV-2 and variants of concern. Science $\mathbf{0}$, eabm0829.

519 31. Dan, J. M. et al. Immunological memory to SARS-CoV-2 assessed for up to 8 months after infection. $520 \quad$ Science 371, eabf4063 (2021).

521 32. Gazit, S. et al. Comparing SARS-CoV-2 natural immunity to vaccine-induced immunity: reinfections versus breakthrough infections. 2021.08.24.21262415 doi:10.1101/2021.08.24.21262415.

33. Reynolds, C. J. et al. Prior SARS-CoV-2 infection rescues B and T cell responses to variants after first vaccine dose. Science 372, 1418-1423 (2021).

34. Wang, Z. et al. mRNA vaccine-elicited antibodies to SARS-CoV-2 and circulating variants. Nature $592,616-622(2021)$.

35. Goel, R. R. et al. Distinct antibody and memory B cell responses in SARS-CoV-2 naïve and recovered individuals after mRNA vaccination. Sci. Immunol. 6, eabi6950 (2021).

36. Saadat, S. et al. Binding and Neutralization Antibody Titers After a Single Vaccine Dose in Health Care Workers Previously Infected With SARS-CoV-2. JAMA 325, 1467-1469 (2021).

37. Cho, A. et al. Anti-SARS-CoV-2 receptor binding domain antibody evolution after mRNA vaccination. Nature 1-9 (2021) doi:10.1038/s41586-021-04060-7.

39. Khoury, D. S. et al. Neutralizing antibody levels are highly predictive of immune protection from symptomatic SARS-CoV-2 infection. Nat. Med. 27, 1205-1211 (2021).

40. Corman, V. M. et al. Detection of 2019 novel coronavirus (2019-nCoV) by real-time RT-PCR.

Eurosurveillance 25, 2000045 (2020).

41. SARS-CoV-2 Immunoassay | Abbott Core Laboratory. https://www.corelaboratory.abbott/us/en/offerings/segments/infectious-disease/sars-cov-2. 
$543 \quad 42 . \quad$ DiaSorin’s LIAISON ${ }^{\circledR}$ SARS-CoV-2 Diagnostic Solutions | DiaSorin.

544 https://www.diasorin.com/en/immunodiagnostic-solutions/clinical-areas/infectious-diseases/covid-19.

545 43. Elecsys ${ }^{\circledR}$ Anti-SARS-CoV-2. https://diagnostics.roche.com/global/en/products/params/elecsys-anti546 sars-cov-2.html. 


\section{Supplementary Files}

This is a list of supplementary files associated with this preprint. Click to download.

- Supplementarymaterialsfinal.pdf 